\title{
Paternal and maternal support on children's weekday and weekend moderate-to-vigorous physical activity: a cross-sectional study
}

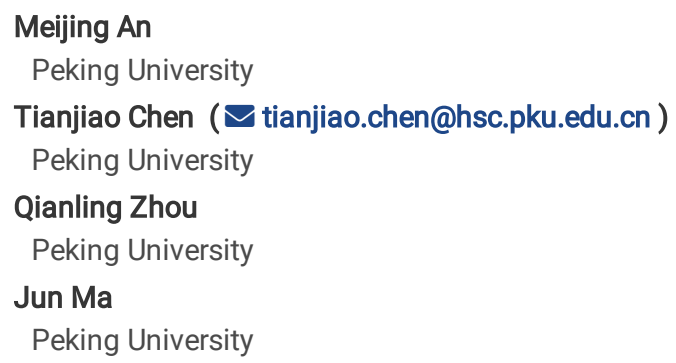




\section{Abstract}

Background: Most studies about parental support behaviors for physical activity (PA) on children's moderate-to-vigorous intensity physical activity (MVPA) were conducted in developed countries, and most of them focused on mother's or parent's (with no differentiation between father's or mother's) support behaviors. Besides, children's MVPA time interval was not differentiated adequately. This paper aimed at investigating the associations between paternal and maternal support behaviors for PA, and children's MVPA on weekdays, weekends and the whole week in China.

Methods: Cross-sectional data of 517 father-child dyads and 1,422 mother-child dyads were analyzed. Children's consecutive 7-day PA diary were recorded to assess their MVPA time, and MVPA time on weekdays, weekends and the whole week were further calculated, respectively. Father or mother completed a questionnaire on their support behaviors for children's PA. Multivariate logistic regression was conducted to investigate the independent effect of paternal and maternal support behaviors for PA on children's meeting MVPA recommendation on weekdays, weekends and the whole week, respectively.

Results: The proportion of children's meeting MVPA recommendation on weekends (37.8\%) was significantly lower than that on weekdays (62.8\%). Higher paternal (OR[95\%Cl]:1.098[1.009,1.195]) and maternal (OR[95\%Cl]:1.076[1.021,1.134]) total scores of support behaviors for PA were associated with children's higher odds of meeting MVPA recommendation on weekends, after controlling for covariates. Paternal sharing PA knowledge with child was significantly associated with children's meeting MVPA recommendation on weekends (OR[95\%CI]:1.319[1.055,1.649]), and marginally associated with children's meeting MVPA recommendation on weekdays (OR[95\% $\mathrm{Cl}]: 1.220[0.974,1.528], P=0.084)$ and on the whole week (OR[95\%Cl]:1.218[0.977,1.519], $P=0.080$ ). Maternal reserving PA time for child was associated with children's higher likelihood of meeting MVPA recommendation on weekdays (OR[95\%Cl]:1.160[1.025,1.313]), weekends (OR[95\%Cl]:1.241[1.097,1.403]) and the whole week

(OR[95\%Cl]:1.214[1.076,1.369]), respectively.

Conclusions: On weekends, paternal and maternal total support behaviors for PA deserves more practices for promoting children's MVPA. To promote children's MVPA on weekdays, weekends and the whole week, father's sharing PA knowledge with child and mother's reserving PA time for child are recommended. Longitudinal researches are needed to verify the effect of paternal and maternal support behaviors on children's MVPA.

\section{Background}

Physical activity (PA), especially for moderate-to-vigorous intensity physical activity (MVPA), is beneficial to children's health. Studies have demonstrated that children's active MVPA could on one hand reduce the risk of obesity[1] and diabetes[2], and on the other hand enhance bone[3] and mental health[4] of children. The World Health Organization (WHO)[5] recommended that children and adolescents should accumulate at least 60 minutes of MVPA daily, with similar guidelines in China[6], USA[7], Canada[8] and Australia[9]. However, substantial percentages of children and adolescents do not meet recommendations of physical activity. The percentage of 13- to 15-year-old adolescents in 105 countries who engaged in $<60$ minutes of MVPA daily has increased to $80.3 \%$ reported in 2012[10]. In China, only $9.4 \%$ boys and $1.9 \%$ girls met the MVPA recommendation, according to the report by Wang $C$ in 2013[11]. Furthermore, children were less active at weekends than weekdays[11, 12]. Some modifiable factors may be benefit for children's active PA and healthy growth.

Ecological model, a multilevel model, was applied to determine the correlates of physical activity extensively[13]. In the interpersonal level, support (e.g. parental encouragement for PA, financial support for PA, parental instrumental and emotional support for PA) from family members influenced children's physical activity $[13,14]$. Meta-analysis retrieving publications to 2014 showed that the association between parental support and child PA was moderate in size $(r=0.38)[15]$. Parental encouragement was moderately associated with child PA $(r=0.34,95 \% \mathrm{Cl}: 0.25-0.41)[15]$. Some studies in recent years had also showed similar results. A large sample investigation in China showed that parental encouragement and financial support for PA were positively associated with a high level of student MVPA participation[16]. Two American studies showed that parental instrumental and emotional support for PA[17] or children's and mother's perceptions of parental support for PA[18] were significantly associated with children's PA. However, in most studies, parental respondents were mothers[15]. This may be associated with the traditional gender roles, mother is usually in charge of private sphere (e.g. home and family), while father is responsible for public sphere (e.g. education and politics)[19].

With shifting of gender roles, men have become more accepting of gender equality in caring for children[20,21]. Several studies showed that there are differences between paternal and maternal support behaviors for PA[22, 23]. While mothers tend to play a larger role in the logistical planning of physical activity, fathers are more likely than mothers to engage in modeling physical[22, 24]. There were only a few studies examined the relationship of paternal and maternal support of physical activity with children's physical activity in developed countries. A study conducted among American preschool children revealed that maternal and paternal support for PA were positively associated with child PA ( $r=0.37$, and $r=0.36$, respectively)[25]. A study in Japan showed maternal support behavior such as watching their child's sports was associated with children's high percentage of MVPA. However, this study failed to find associations between children's MVPA and paternal support[26]. A study in Australia showed that maternal role modeling or paternal reinforcement of PA was positively associated with boys' after school or weekend MVPA[27]. Most studies about parental support behaviors were limited to developed countries such as United States and Australia[15]. The associations between paternal or maternal support behaviors and children's MVPA were inconsistent among different countries. Considering the potential cultural differences of parenting practices[28], an investigation into the influence of paternal or maternal support behaviors on children's MVPA is needed to be conducted in China. 
To our knowledge, only a few studies presenting parental support behaviors on children's MVPA of different time interval. Morrissey reported that, in Germany, increased in family support resulted in an increased in total time of MVPA and time in MVPA after 3 pm on weekdays[29]. A study in Australia found that different support aspects from mother or father were positively associated with boys' MVPA after school or weekend[27]. A study in Canada showed that increased parental encouragement was positively associated with children's PA on weekdays and girls' PA on weekend days. Increased parental care was positively associated with boys' PA on weekend days[30]. Differentiating time interval could help policy makers and researchers to develop strategies to promote children's physical activity. In China, only one relevant study presented that parental support was significantly associated with children's MVPA on the whole week; however, this study failed to differentiate children's MVPA on weekdays or weekend days [16].

In all, although it is very likely that parental support exerts important role in children's MVPA, there are areas unexplored. Firstly, fathers' physical activity support toward school-aged children was less concerned, especially in China. Secondly, parental support behaviors for PA on children's different MPVA time intervals (e.g. weekdays and weekends) were not studied adequately in neither China nor other countries. As a result, the present study aimed to investigate the associations between paternal and maternal support for physical activity and children's MVPA time, and to differentiate the association of parental support on children and adolescents' MVPA behaviors on weekdays and weekends. The hypothesis is that paternal and maternal support behaviors for PA are associated with children's MVPA, and there are different effects on weekdays and on weekends.

\section{Methods}

\section{Participants}

This was a cross-sectional study conducted between October and December 2012 in Fangshan District, Beijing, China. The method of stratified cluster sampling was applied. The students from grade 2 to 5 in primary schools, grade 7 to 8 in middle schools and their parents were recruited from 16 schools (four urban middle schools, four urban primary schools, four rural middle schools, and four rural primary schools). Students with serious organic diseases (e.g. heart, lung, liver, kidney, and other related diseases), or abnormal body development, or cause-based obesity (e.g. endocrine disease or drug side effect) were excluded. There were 3,441 questionnaires distributed to students and parents, respectively. There were 2,670 complete and valid questionnaires received from students, and the paired questionnaire from parents were excluded if parental support behaviors were not filled in the questionnaire. There were finally 1939 valid parental questionnaires, including 517 completed by fathers and 1,422 by mothers. Ethical approval was obtained from the Ethical Committee of Peking University. Written informed consent were obtained from all participating children and their parents before any assessment.

\section{Measures}

\section{MVPA}

Children and adolescents' physical activity was assessed using consecutive 7-day physical activity diary, including five weekdays and two weekend days. Students' activities and time length were required to be recorded at the end of each day. Combined with children's daily activities and adapted from previous study[31], the diary contents were designed by a group of experts. The items included (1) How to go to school? (2) How to leave school? The answers for these two items are no schooling, walking, bicycling, and taking a car. Those students with walking, bicycling, and taking a car were required to record time length. (3) Did you walk outside today? E.g. shopping, visiting relatives or friends, going to a park. (4) Did you do homework today? (5) Did you watch TV today? (6) Did you play computer or e-games today? (7) Did you have PE class today? (8) Besides PE class, did you do MVPA in school today? (9) Did you do MVPA out of school. MVPA in item (8) and (9) referred to those activities that make you sweat, gasp or feel a little tired or very tired, such as running, playing football, cycling, dancing and so on. The answers for item (3) (9) were yes or no. If yes, the time length was required to be recorded.

In this study, bicycling to school or leaving school, having PE class, doing MVPA in school and doing MVPA out of school were regarded as MVPA. Time length of each activity were summed as every day total MVPA time. The dairy is validated if it was recorded more than or equal to three days on weekdays and more than or equal to one day on weekends[27]. The mean time of MVPA on weekdays equals to total validated MVPA time from Monday to Friday dividing by the number of validated days. The mean time of MVPA in weekend days equals to total validated MVPA time from Saturday to Sunday dividing by number of validated days. The mean time of every day equals to total validated MVPA time from Monday to Sunday dividing by number of validated days. In analysis, the daily continuous MVPA time on weekdays, on weekends, on the whole week were transformed into categorical labels according to physical activity recommendation released by the WHO[5] and by China[6]. The cut-off value is 60 minutes. Namely, the labels of daily MVPA time on weekdays, weekends and the whole week were $\geq 60$ minutes (i.e. meeting MVPA recommendation) and 600 minutes (i.e. not meeting MVPA recommendation), respectively.

\section{Parental support for physical activity}

Questionnaire data to evaluate parental support behaviors for children's physical activity were collected. Parental support behaviors were evaluated by the following three questions on a 5-point Likert scale from never (1) to always (5). Q1, "As a parent, do you share your knowledge of physical activity/exercise with your child?" (marked as share PA knowledge with child below); Q2, "Do you tell your child and help your child cultivate good exercise habits after you requiring knowledge of physical activity/exercise?” (marked as cultivate child's PA habits below); Q3, “Do you reserve time 
for your child to participate physical activity/exercise?" (marked as reserve PA time for child below). In the current sample, the Cronbach's alpha of physical activity support was 0.762 and 0.778 for fathers and mothers, respectively. The sum of these three variables was used as a measure of the paternal or maternal total score of support behaviors.

\section{Covariates}

Gender, grade, school name were collected from the physical activity diary; and information on children's district and school stage was obtained. Children's age was calculated according to their birthdate and examination date recorded. Children's weight and height were measured objectively by trained postgraduates, and BMI $\left(\mathrm{kg} / \mathrm{m}^{2}\right)$ was further calculated. Children's weight status (normal or wasting, overweight or obesity) was defined based on the age- and gender-specific BMI cutoffs according to the standards of screening for overweight and obesity among school-aged children and adolescents, as published in China in 2018[32].

Paternal or maternal age, education level, height and weight were collected from the questionnaire. Paternal or maternal BMI was calculated the same as children's. Paternal or maternal weight status (normal or wasting, overweight or obesity) was classified according to Chinese criteria of weight for adults[33].

\section{Data analysis}

General description for normally distributed variables was presented as means and standard deviations. Categorical data were summarized by frequency and percentage. Differences in proportions were tested by Chi-square test for independent sample comparation or McNemar test for matched sample comparation. Differences in means for continuous variables were compared using student's t-test. Multivariate logistic regression was used to examine the association between paternal or maternal support behavior score and children's meeting MVPA recommendation on weekdays, on weekends and on the whole week. Owing to correlation between parental BMI and children's BMI ( $r=0.171$ between father and children, and $r=0.220$ between mother and children) and correlation between parental age and children's school stage ( $t=-6.622, P<0.001$ between father and children; $t=-14.204, P<0.001$ between mother and children), logistic regressions were conducted after controlling for children's school stage, gender, district, weight status, and paternal or maternal educational level. All analyses were performed by SPSS (version 22.0), with Pख0.05 considered as statistically significant.

\section{Results}

Children's characteristics are illustrated in Table 1. The mean age was $11.35 \pm 2.17$ years. Overweight or obese children accounted for $34.6 \%$. There were $57.0 \%$ children met MVPA recommendation on the whole week, and the corresponding figures between weekdays (62.8\%) and weekends (37.8\%) were significantly different $(P<0.001)$. Characteristics of children in father-child dyads and in mother-child dyads were similar in age, schooling stage, weight status, daily weekday MVPA, daily weekend MVPA and daily MVPA.

Paternal and maternal mean ages were $38.83 \pm 4.29$ and $36.85 \pm 4.55$ years, respectively. As shown in Table $2,61.7 \%$ fathers and $44.4 \%$ mothers were overweight or obesity. Father's total support scores were higher than mother's. The percentage distribution of three support behaviors (i.e. share PA knowledge with child, cultivate child's PA habits, and reserve PA time for child) in father sample and in mother sample were similar.

Children from rural districts were more likely to meet MVPA recommendation on weekdays and on the whole week than those from urban districts. Primary school students were less likely to meet MVPA recommendation on weekdays but more likely to meet recommendation on weekends, in comparison to middle school students. Normal weight or wasting students were more likely to meet MVPA recommendation on weekdays than overweight or obese counterparts. Paternal and maternal educational level, and maternal weight status were associated with children's MVPA, respectively (Supplementary table S1).

Univariate association between paternal and maternal support behaviors and children's MVPA are shown in Table 3 and Table 4, respectively. Paternal total scores of support behaviors and paternal support behavior of sharing PA knowledge with child were higher in children meeting MVPA recommendation on weekends than those who did not meet recommendation. Maternal total scores of support behaviors were higher in children meeting MVPA recommendation on weekends, as well as on the whole week. Maternal score of reserving PA time for children was positively associated with children's meeting MVPA recommendation on weekdays, weekends and on the whole week.

As shown in Table 5, after controlling for covariates, higher paternal total support score was associated with children's meeting MVPA recommendation on weekends $(\mathrm{OR}=1.098,95 \% \mathrm{Cl}$ :1.009-1.195). Paternal sharing PA knowledge with child was significantly associated with children's meeting MVPA recommendation on weekends (OR=1.319, 95\% Cl:1.055-1.649), marginally significantly associated meeting recommendation on weekdays ( $\mathrm{OR}=1.220,95 \% \mathrm{Cl}: 0.974-1.528, P=0.084)$ and on the whole week (OR=1.218, 95\% Cl:0.977-1.519, $P=0.080)$. Similarly, mother's total support score was positively associated with meeting MVPA recommendation on weekends. Besides, mother's cultivating child's PA habits (OR=1.171, 95\% Cl:1.024-1.339) was related to children's meeting recommendation on weekends. Higher score of maternal reserving PA time for child was associated with higher likelihood of children's meeting MVPA recommendation on weekdays (OR=1.160, 95\% Cl:1.025-1.313), weekends $(\mathrm{OR}=1.241,95 \% \mathrm{Cl}: 1.097-1.430)$ and on the whole week (OR=1.214, 95\% Cl:1.076-1.369), respectively. 


\section{Discussion}

Our study examined the relationships between paternal and maternal support behaviors for PA and children's MVPA on weekdays, on weekends and on the whole week in China. It was found that paternal and maternal total score of support behaviors were associated with children's higher odds of meeting MVPA recommendation on weekends. Maternal cultivating child's PA habits was related to children's meeting MVPA recommendation on weekends. Paternal sharing PA knowledge with child and maternal reserving PA time for child was positively associated with children's meeting recommendation on weekdays, weekends and on the whole week.

Our study found that paternal and maternal total scores of support behaviors for PA were associated with children's higher odds of meeting MVPA recommendation on weekends. Our result is in consistent with previous study that family support may exert great influences on promoting adolescents' MVPA positivity [34]. For each unit increase in family support, daily weekday MVPA was increased by 0.86 min/d, while weekend MVPA was increased by $1.28 \mathrm{~min} /$ day [34]. There are more PA opportunities within school settings on weekdays, such as PE class, and school-level PA support works more on children's MVPA[35,36]. In contrast, children's movements at weekends are likely to take place at home or at community, and guided by their parents [30]. A study also showed that increased parental encouragement was positively associated with girls' physical activity on weekend days; and increased parental care was associated with boys' physical activity on weekend days[30]. Weekends may be the optimal time for parents to provide support behaviors to their children's MVPA. Our study revealed that only $37.8 \%$ children met MVPA recommendation on weekends, which was significantly lower than the proportion on weekdays. Our result is in accordance with the undesiring trend in the world[12, 37].

Our study indicated that children's higher odds of meeting MVPA recommendation was associated with maternal cultivating PA habits and reserving PA time for child, but not maternal sharing knowledge with child. Our findings are supported by previous evidence that mothers' encouragement may not always promote children's PA[38]. Some Chinese mothers are characterized as nagging[39]. They might be considered as bothersome when they share PA knowledge to their children, and further having negative influence on children's MVPA[40]. In contrast, direct support behaviors such as cultivating children's PA habits and reserving PA time for children may be positive venues for mothers to guide children performing more active MVPA. Similarly, Tanaka et al. reported that children whose mothers watched children's sports events had higher MVPA level than those whose mothers did not watch these events[26]. Therefore, mothers' practicing direct support behaviors may be helpful to their children's MVPA level.

With societal shift in family structure, fathers have become the primary care providers [41]. A systematic review reported that few studies had examined the effect of father on child physical activity, and the relationship between father's influence and children's PA remained unclear. Our study that paternal sharing PA knowledge with child being positively related to children's MVPA have added evidence to the literature. Likewise, a study in Japan failed to demonstrate significant associations between paternal support and children's MVPA[26]. Moreover, fathers' modeling of MVPA has been shown to be associated with higher level of physical activity in their children[22, 23]. The role of paternal PA modeling had not been examined in our study and might be considered in future investigation.

Our study is novel in the differentiation between paternal and maternal influence on children's MVPA, and the observation of children's MVPA in different time intervals which detected the importance of paternal and maternal support on children's MVPA on weekends. However, our study has some limitations. First, our study was designed as cross-sectional, and the causal inference could thus not be identified. The pattern of associations may also reflect the possibility that highly active children elicited higher levels of support from their parents. Longitudinal study is needed to determine the direction of association between parental PA support and children's physical activity. Second, only three aspects of parental support behaviors were included in our study. Other aspects of support behaviors such as PA financial support, PA accompanying, PA modeling have not been examined. Developing and using more comprehensive support behavior scale is warranted in future studies. Third, although consecutive 7-day physical activity diary can be used to avoid recall bias and mirror children's physical activity pattern in a cycle of seven days[42], its accuracy was inferior to accelerometer. Nevertheless, physical activity diary is more feasible than accelerometer in our study, considering our relatively large sample size. Moreover, children's behaviors and time were recorded at the end of every day can also reduce parents' expectation bias. Fourth, in an attempt to reduce respondent burden, only one of parent (father or mother) was investigated in our study. The results showed that the different associations between maternal and paternal support behaviors with children's MVPA might not be statistically significant in our study. Further studies conducted among both mother and father are needed to confirm the significance of different associations.

\section{Conclusion}

Parental total support behaviors for PA appear to play a critical role in promoting children's MVPA on weekends, implying that appropriate intervention targeting parents may works to counteract the ascending trend towards inactivity in children, especially on weekend days. Maternal direct support behaviors such as cultivating child's PA habits and reserving PA time for child, and paternal verbal support behavior such as sharing PA knowledge with child had influences on their children's MVPA. Tailored intervention strategies should be considered separately between father and mother. Longitudinal researches are warranted to verify the associations between paternal and maternal support behaviors for PA and children's MVPA.

\section{Abbreviations}

PA: Physical Activity; MVPA: Moderate-to-Vigorous intensity Physical Activity; BMI: Body Mass Index. Cl: Credible Interval. WHO: World Health Organization. 


\section{Declarations}

\section{Acknowledgements}

All authors are grateful to all children, their fathers, and their mothers. We also wish to thank teachers, local education and health staff, and research assistants involved.

\section{Funding}

This study was funded by special research grant for non-profit public service of the Ministry of Health of China (Grant No. 201202010). The funding body had no role in the design of the study, in the collection, analysis, and interpretation of data, or in writing the manuscript.

\section{Availability of data and materials}

The dataset for the present study is available from the corresponding author upon reasonable request.

\section{Authors' contributions}

MA cleaned and analyzed the data, interpreted the results and drafted the paper. TC conceived and designed the study, conducted the field work, and provide guidance in paper writing. JM conceived and designed the study, secured funding, oversaw the study's implementation, and commented on the paper. QZ provided critical comments on this paper. All authors critically revised the paper, and read and approved the final manuscript.

\section{Competing interests}

The authors declare that they have no competing interests.

\section{Ethics approval and consent to participate}

Ethical approval for present study was obtained from the Ethical Committee of Peking University. All participating children and parents provided written informed consent.

\section{Consent for publication}

Not applicable.

\section{Author details}

1 Institute of child and adolescent health, School of Public Health, Peking University, No.38 Xueyuan Road, Haidian District, Beijing, China, 100191.

2 Department of Maternal and Child Health, School of Public Health, Peking University, No.38 Xueyuan Road, Haidian District, Beijing, China, 100191.

\section{References}

1. Poitras VJ, Gray CE, Borghese MM, Carson V, Chaput JP, Janssen I, et al. Systematic review of the relationships between objectively measured physical activity and health indicators in school-aged children and youth. Appl Physiol Nutr Metab. 2016;41(6 Suppl 3):S197-239.

2. Ungethüm K, Jolink M, Hippich M, Lachmann L, Haupt F, Winkler C, et al. Physical activity is associated with lower insulin and C-peptide during glucose challenge in children and adolescents with family background of diabetes. Diabet Med. 2019;36(3):366-75.

3. Osborn W, Simm P, Olds T, Lycett K, Mensah FK, Muller J, et al. Bone health, activity and sedentariness at age 11-12years: Cross-sectional Australian population-derived study. Bone. 2018;112:153-60.

4. Donnelly JE, Hillman CH, Greene JL, Hansen DM, Gibson CA, Sullivan DK, et al. Physical activity and academic achievement across the curriculum: Results from a 3-year cluster-randomized trial. Prev Med. 2017;99:140-5.

5. World Health Organization: Global recommendations on physical activity for health. http://www.who.int/dietphysicalactivity/factsheet_recommendations/en/ (2010). Accessed 6/7 2020.

6. Zhang Y, Ma S, Chen C, Liu S, Zhang C, Cao Z, et al. [Physical activity guideline for Chinese children and adolescents]. Chin J Evid Based Pediatr 2017;12(6):401-9.

7. Office of Disease Prevention and Health Promotion: Physical activity guidelines for Americans:2nd edition. https://health.gov/paguidelines/second-edition/ (2018). Accessed 6/7 2020.

8. Tremblay MS, Carson V, Chaput JP, Connor Gorber S, Dinh T, Duggan M, et al. Canadian 24-hour movement guidelines for children and youth: An integration of physical activity, sedentary behaviour, and sleep. Appl Physiol Nutr Metab. 2016;41(6 Suppl 3):S311-27.

9. Australian Government Department Of Health: Australia's physical activity and sedentary behaviour guidelines and the Australian 24-hour movement guidelines. https://www1.health.gov.au/internet/main/publishing.nsf/content/health-pubhlth-strateg-phys-act-guidelines/ (2019). 
Accessed 6/7 2020.

10. Hallal PC, Andersen LB, Bull FC, Guthold R, Haskell W, Ekelund U. Global physical activity levels: surveillance progress, pitfalls, and prospects. Lancet. 2012;380(9838):247-57.

11. Wang C, Chen P, Zhuang J. A national survey of physical activity and sedentary behavior of Chinese city children and youth using accelerometers. Res Q Exerc Sport. 2013;84 Suppl 2:S12-28.

12. Brooke HL, Corder K, Atkin AJ, van Sluijs EM. A systematic literature review with meta-analyses of within- and between-day differences in objectively measured physical activity in school-aged children. Sports Med. 2014;44(10):1427-38.

13. Bauman AE, Reis RS, Sallis JF, Wells JC, Loos RJ, Martin BW. Correlates of physical activity: why are some people physically active and others not? Lancet. 2012;380(9838):258-71.

14. Kellou N, Sandalinas F, Copin N, Simon C. Prevention of unhealthy weight in children by promoting physical activity using a socio-ecological approach: what can we learn from intervention studies? Diabetes Metab. 2014;40(4):258-71.

15. Yao CA, Rhodes RE. Parental correlates in child and adolescent physical activity: a meta-analysis. Int J Behav Nutr Phys Act. $2015 ; 12: 10$.

16. Liu Y, Zhang Y, Chen S, Zhang J, Guo Z, Chen P. Associations between parental support for physical activity and moderate-to-vigorous physical activity among Chinese school children: A cross-sectional study. J Sport Health Sci. 2017;6(4):410-5.

17. Heredia NI, Ranjit N, Warren JL, Evans AE. Association of parental social support with energy balance-related behaviors in low-income and ethnically diverse children: a cross-sectional study. BMC Public Health. 2016;16(1):1182.

18. Forthofer M, Dowda M, Mclver K, Barr-Anderson DJ, Pate R. Associations between maternal support and physical activity among 5th grade students. Matern Child Health J. 2016;20(3):720-9.

19. Louise A. Tilly, Joan W. Scott. Women, work and family. New York: Holt, Rinehart \& Winston; 1978.

20. Gregory SK. The unfinished revolution: coming of age in a new era of gender, work and family. Community Work \& Family. 2011;18(3):1-2.

21. Solomon-Moore E, Toumpakari Z, Sebire SJ, Thompson JL, Lawlor DA, Jago R. Roles of mothers and fathers in supporting child physical activity: a cross-sectional mixed-methods study. BMJ Open. 2018;8(1):e019732.

22. Davison KK, Cutting TM, Birch LL. Parents' activity-related parenting practices predict girls' physical activity. Med Sci Sports Exerc. 2003;35(9):1589-95.

23. Edwardson CL, Gorely T. Activity-related parenting practices and children's objectively measured physical activity. Pediatr Exerc Sci. 2010;22(1):105-13.

24. Lloyd AB, Lubans DR, Plotnikoff RC, Collins CE, Morgan PJ. Maternal and paternal parenting practices and their influence on children's adiposity, screen-time, diet and physical activity. Appetite. 2014;79:149-57.

25. Schoeppe S, Trost SG. Maternal and paternal support for physical activity and healthy eating in preschool children: a cross-sectional study. BMC Public Health. 2015;15:971.

26. Tanaka C, Okuda M, Tanaka M, Inoue S, Tanaka S. Associations of physical activity and sedentary time in primary school children with their parental behaviors and supports. Int J Environ Res Public Health. 2018;15(9):1995.

27. Cleland V, Timperio A, Salmon J, Hume C, Telford A, Crawford D. A longitudinal study of the family physical activity environment and physical activity among youth. Am J Health Promot. 2011;25(3):159-67.

28. Liu M, Guo F. Parenting practices and their relevance to child behaviors in Canada and China. Scand J Psychol. 2010;51(2):109-14.

29. Morrissey JL, Janz KF, Letuchy EM, Francis SL, Levy SM. The effect of family and friend support on physical activity through adolescence: a longitudinal study. Int J Behav Nutr Phys Act. 2015;12:103.

30. Vander Ploeg KA, Kuhle S, Maximova K, McGavock J, Wu B, Veugelers PJ. The importance of parental beliefs and support for pedometermeasured physical activity on school days and weekend days among Canadian children. BMC Public Health. 2013;13:1132.

31. Wen LM, van der Ploeg HP, Kite J, Cashmore A, Rissel C. A validation study of assessing physical activity and sedentary behavior in children aged 3 to 5 years. Pediatr Exerc Sci. 2010;22(3):408-20.

32. National Health Commission of the People's Republic of China. [Screening for overweight and obesity among school-age children and adolescents]. 2018.

33. National Health Commission of the People's Republic of China. [Criteria of weight for adults]. 2013.

34. Baskin ML, Thind H, Affuso O, Gary LC, LaGory M, Hwang SS. Predictors of moderate-to-vigorous physical activity (MVPA) in African American young adolescents. Ann Behav Med. 2013;45 Suppl 1(0 1):S142-50.

35. Taylor SL, Curry WB, Knowles ZR, Noonan RJ, McGrane B, Fairclough SJ. Predictors of segmented school day physical activity and sedentary time in children from a northwest England low-income community. Int J Environ Res Public Health. 2017;14(5):534.

36. Wang L, Tang Y, Luo J. School and community physical activity characteristics and moderate-to-vigorous physical activity among Chinese school-aged children: A multilevel path model analysis. J Sport Health Sci. 2017;6(4):416-22.

37. Nader PR, Bradley RH, Houts RM, McRitchie SL, O'Brien M. Moderate-to-vigorous physical activity from ages 9 to 15 years. JAMA. 2008;300(3):295-305. 
38. Lopez NV, Yang CH, Belcher BR, Margolin G, Dunton GF. Within-subject associations of maternal physical activity parenting practices on children's objectively measured moderate-to-vigorous physical activity. J Pediatr Psychol. 2019;44(3):300-10.

39. Mo G, Zhang L. [Interpretation of speech behaviour of nagging based on the Theory of Cognitive Dissonance]. Journal of XuZhou Institute of Architectural Technology. 2007;7(4):59-62.

40. Robbins LB, Talley HC, Wu TY, Wilbur J. Sixth-grade boys' perceived benefits of and barriers to physical activity and suggestions for increasing physical activity. J Sch Nurs. 2010;26(1):65-77.

41. Arlinghaus KR, Johnston CA. Engaging fathers in the promotion of healthy lifestyle behaviors. Am J Lifestyle Med. 2017;11(3):216-9.

42. Rowlands AV, Gomersall SR, Tudor-Locke C, Bassett DR, Kang M, Fraysse F, et al. Introducing novel approaches for examining the variability of individuals' physical activity. J Sports Sci. 2015;33(5):457-66.

\section{Tables}

\begin{tabular}{|llll|}
\hline Table 1 Characteristics of children & & & \\
\hline Variables & $\begin{array}{l}\text { All children } \\
(\mathrm{n}=1939)\end{array}$ & $\begin{array}{l}\text { Children in father-child dyads } \\
(\mathrm{n}=517)\end{array}$ & $\begin{array}{l}\text { Children in mother-child dyads } \\
(\mathrm{n}=1422)\end{array}$ \\
\hline Age(years), mean(SD) & $11.35 \pm 2.17$ & $11.41 \pm 2.13$ & $11.32 \pm 2.18$ \\
\hline Male sex ${ }^{\mathrm{a}} \mathrm{n}(\%)$ & $959(49.5)$ & $288(55.7)$ & $671(47.2)$ \\
\hline Urban district ${ }^{\mathrm{a}} \mathrm{n}(\%)$ & $895(46.2)$ & $202(39.1)$ & $693(48.7)$ \\
\hline Primary school stage,n(\%) & $934(48.2)$ & $250(48.4)$ & $684(48.1)$ \\
\hline Overweight or obesity, $\mathrm{n}(\%)$ & $670(34.6)$ & $167(32.3)$ & $503(35.4)$ \\
\hline Daily weekday MVPA $\geq 60$ min, $\mathrm{n}(\%)$ & $1218(62.8)$ & $325(62.9)$ & $893(62.8)$ \\
\hline Daily weekend MVPA $\geq 60$ min, $\mathrm{n}(\%)$ & $732(37.8)$ & $296(37.9)$ & $536(37.7)$ \\
\hline Daily MVPA $\geq 60$ min, $\mathrm{n}(\%)$ & $1106(57.0)$ & $303(58.6)$ & $803(56.5)$ \\
\hline
\end{tabular}

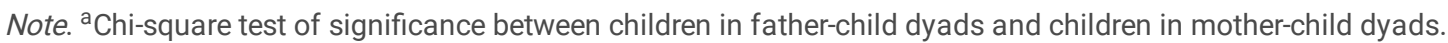




\begin{tabular}{|c|c|c|}
\hline Variables & Father & Mother \\
\hline $\mathrm{Age}^{\star}, \geq 40$ years old & 197(41.7) & $313(24.5)$ \\
\hline \multicolumn{3}{|l|}{ Education level* } \\
\hline middle school or below & $254(53.8)$ & $605(47.4)$ \\
\hline senior or junior school & 179(37.9) & $523(41.0)$ \\
\hline university or above & $39(8.3)$ & $148(11.6)$ \\
\hline Overweight or obesity* & 291(61.7) & $566(44.4)$ \\
\hline Total support scores ${ }^{a}$ & $10.21 \pm 2, .28$ & $9.93 \pm 2.21$ \\
\hline \multicolumn{3}{|c|}{ Share PA knowledge with child } \\
\hline never & $5(1.0)$ & $15(1.1)$ \\
\hline Rarely & $59(11.4)$ & $155(10.9)$ \\
\hline Sometime & $185(35.8)$ & $615(43.2)$ \\
\hline Often & 205(39.6) & $494(34.7)$ \\
\hline Always & $63(12.2)$ & $143(10.1)$ \\
\hline \multicolumn{3}{|l|}{ Cultivate child's PA habits } \\
\hline Never & $4(0.8)$ & $10(0.7)$ \\
\hline Rarely & 71(13.7) & $160(11.3)$ \\
\hline Sometime & 179(34.6) & $583(41.0)$ \\
\hline Often & 201(38.9) & $517(36.3)$ \\
\hline Always & $62(12.0)$ & 152(10.7) \\
\hline \multicolumn{3}{|l|}{ Reserve PA time for child } \\
\hline Never & $14(2.7)$ & $33(2.3)$ \\
\hline Rarely & 108(20.9) & $387(27.2)$ \\
\hline Sometime & 197(38.1) & $562(39.5)$ \\
\hline Often & $145(28.0)$ & $338(23.8)$ \\
\hline Always & $53(10.3)$ & $102(7.2)$ \\
\hline
\end{tabular}

Note. This table provides frequency and percentage for categorical variables, and provides means and standard deviations for continuous variables.

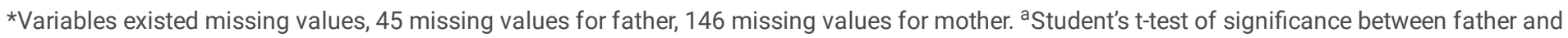
mother.

\begin{tabular}{|c|c|c|c|c|c|c|}
\hline \multirow[t]{3}{*}{ Variables } & \multicolumn{2}{|c|}{ Daily weekday MVPA time } & \multicolumn{2}{|c|}{ Daily weekend MVPA time } & \multicolumn{2}{|c|}{ Daily MVPA time } \\
\hline & $\otimes 60 \mathrm{~min}$ & $\geq 60 \mathrm{~min}$ & $凶 60 \mathrm{~min}$ & $\geq 60 \mathrm{~min}$ & $\triangle 60 \mathrm{~min}$ & $\geq 60 \mathrm{~min}$ \\
\hline & $(n=192)$ & $(n=325)$ & $(n=321)$ & $(n=196)$ & $(n=214)$ & $(n=303)$ \\
\hline Total support scores & $10.10 \pm 2.28$ & $10.26 \pm 2.28$ & $10.03 \pm 2.29$ & $10.48 \pm 2.24$ & $10.07 \pm 2.31$ & $10.30 \pm 2.26$ \\
\hline Share PA knowledge with child & $3.46 \pm 0.90$ & $3.53 \pm 0.87$ & $3.44 \pm 0.89$ & $3.62 \pm 0.87$ & $3.46 \pm 0.91$ & $3.54 \pm 0.86$ \\
\hline Cultivate child's PA habits & $3.46 \pm 0.92$ & $3.49 \pm 0.89$ & $3.42 \pm 0.90$ & $3.57 \pm 0.90$ & $3.46 \pm 0.93$ & $3.49 \pm 0.88$ \\
\hline Reserve PA time for child & $3.18 \pm 1.03$ & $3.25 \pm 0.95$ & $3.17 \pm 0.99$ & $3.30 \pm 0.95$ & $3.15 \pm 1.01$ & $3.27 \pm 0.96$ \\
\hline
\end{tabular}


Note. Bold results refers to significant difference between group $\geq 60 \mathrm{~min}$ and $₫ 60 \mathrm{~min}$ in each time interval.

Table 4 Univariate association between maternal support behaviors and children's MVPA

\begin{tabular}{|c|c|c|c|c|c|c|}
\hline \multirow[t]{2}{*}{ Variables } & \multicolumn{2}{|c|}{ Daily weekday MVPA time } & \multicolumn{2}{|c|}{ Daily weekend MVPA time } & \multicolumn{2}{|c|}{ Daily MVPA time } \\
\hline & $\begin{array}{l}\otimes 60 \mathrm{~min} \\
(\mathrm{n}=529)\end{array}$ & $\begin{array}{l}\geq 60 \mathrm{~min} \\
(\mathrm{n}=893)\end{array}$ & $\begin{array}{l}\triangle 60 \mathrm{~min} \\
(\mathrm{n}=886)\end{array}$ & $\begin{array}{l}\geq 60 \text { min } \\
(n=536)\end{array}$ & $\begin{array}{l}\triangle 60 \mathrm{~min} \\
(\mathrm{n}=619)\end{array}$ & $\begin{array}{l}\geq 60 \mathrm{~min} \\
(\mathrm{n}=803)\end{array}$ \\
\hline Total support scores & $9.80 \pm 2.16$ & $10.01 \pm 2.23$ & $9.80 \pm 2.18$ & $10.15 \pm 2.24$ & $9.77 \pm 2.15$ & $10.06 \pm 2.24$ \\
\hline Share PA knowledge with child & $3.40 \pm 0.84$ & $3.43 \pm 0.86$ & $3.40 \pm 0.84$ & $3.44 \pm 0.87$ & $3.39 \pm 0.85$ & $3.44 \pm 0.86$ \\
\hline Cultivate child's PA habits & $3.43 \pm 0.84$ & $3.47 \pm 0.86$ & $3.41 \pm 0.85$ & $3.52 \pm 0.86$ & $3.42 \pm 0.83$ & $3.48 \pm 0.87$ \\
\hline Reserve PA time for child & $2.97 \pm 0.91$ & $3.12 \pm 0.95$ & $2.99 \pm 0.94$ & $3.19 \pm 0.93$ & $2.96 \pm 0.93$ & $3.14 \pm 0.94$ \\
\hline
\end{tabular}

Note. Bold results refers to significant difference between group $\geq 60 \mathrm{~min}$ and $\$ 60 \mathrm{~min}$ in each time interval.

Table 5 Adjusted association of parental support behaviors and children's MVPA meeting the recommendation

\begin{tabular}{|c|c|c|c|c|c|c|}
\hline \multirow[t]{2}{*}{ Variables } & \multicolumn{2}{|c|}{ Daily weekday MVPA time } & \multicolumn{2}{|c|}{ Daily weekend MVPA time } & \multicolumn{2}{|l|}{ Daily MVPA time } \\
\hline & Father & Mother & Father & Mother & Father & Mother \\
\hline $\begin{array}{l}\text { Total } \\
\text { support } \\
\text { scores }\end{array}$ & $1.053(0.967,1.146)$ & $1.042(0.989,1.098)$ & $1.098(1.009,1.195)$ & $1.076(1.021,1.134)$ & $1.061(0.976,1.154)$ & $1.060(1.007,1.115)$ \\
\hline $\begin{array}{l}\text { Share PA } \\
\text { knowledge } \\
\text { with child }\end{array}$ & $1.220(0.974,1.528)$ & $1.067(0.929,1.224)$ & $1.319(1.055,1.649)$ & $1.069(0.932,1.226)$ & $1.218(0.977,1.519)$ & $1.080(0.944,1.235)$ \\
\hline $\begin{array}{l}\text { Cultivate } \\
\text { child's PA } \\
\text { habits }\end{array}$ & $1.050(0.845,1.306)$ & $1.032(0.903,1.180)$ & $1.213(0.978,1.504)$ & $1.171(1.024,1.339)$ & $1.031(0.833,1.276)$ & $1.078(0.946,1.228)$ \\
\hline $\begin{array}{l}\text { Reserve } \\
\text { PA time } \\
\text { for child }\end{array}$ & $1.084(0.892,1.318)$ & $1.160(1.025,1.313)$ & $1.131(0.933,1.372)$ & $1.241(1.097,1.403)$ & $1.149(0.948,1.392)$ & $1.214(1.076,1.369)$ \\
\hline
\end{tabular}

\section{Supplementary Files}

This is a list of supplementary files associated with this preprint. Click to download.

- 9PAsupportforBMCPHadditionaltable.docx 